\title{
Innovación didáctica: la gestión ambiental como eje transversal en educación primaria y secundaria
}

\section{Teaching innovation: environmental management as a central focus in primary and secondary}

\author{
Álvaro Fernández González' \\ Nancy Hidalgo Dittel ${ }^{2}$ \\ Florangel Villegas Verdú ${ }^{3}$ \\ Jeison Alfaro Aguirre 4 \\ Yanury Chaves Solano \\ Miguel Guevara Agüero ${ }^{6}$
}

I Observatorio del Desarrollo, Universidad de Costa Rica. Teléfono: 25 I I-4878

Correo electrónico: afernandez@odd.ucr.ac.cr

2 Escuela de Ingeniería Agrícola, Tecnológico de Costa Rica. Teléfono: 2550-227I, Correo electronico: nhidalgo@itcr.ac.cr

3 Escuela de Ciencias Exactas y Naturales, Universidad Estatal a Distancia (ECEN-UNED). Teléfono: 8781-3194.

Correo electrónico: fvillegas@uned.ac.cr

4 Escuela de Educación Técnica, Tecnológico de Costa Rica. Teléfono: 2550-2376. Correo electrónico: jalfaroa03@gmail.com

5 Consultora Universidad de Costa Rica-Tecnológico de Costa Rica. Teléfono: 8923-1941 Correo electrónico: yanury.chaves@yahoo.com

6 Escuela de Educación Técnica, Tecnológico de Costa Rica.

Este artículo cuenta con el aval de la Vicerrectoría de Investigación y Extensión del Tecnológico de Costa Rica 


\section{Resumen}

El objetivo de este proyecto es brindar, a docentes, herramientas teóricas y prácticas relevantes, con actividades curriculares o extracurriculares de gestión ambiental en primaria, secundaria o educación diversificada, a fin de que puedan desarrollar y evaluar propuestas sistemáticas de innovación didáctica en este campo.

Se procura crear las condiciones para que centros educativos públicos del sur de Costa Rica incorporen un componente que estimule el desarrollo de destrezas y habilidades para la participación ciudadana en la gestión ambiental a escala local (cantonal o distrital). El proyecto inició con la Dirección Regional de Educación de Pérez Zeledón y, en el 2012, el proyecto se extendió a la Dirección Regional de Educación Grande de Térraba (la cual abarca los cantones de Buenos Aires de Puntarenas y Osa) por solicitud expresa de esta dirección regional.

Se aplicó un enfoque de extensión docente y educación continua para fortalecer el currículo de centros educativos con innovaciones didácticas en el tema transversal de cultura ambiental para el desarrollo sostenible.

La estrategia fundamental del proyecto se ha centrado en el desarrollo de un curso modular de "Innovación educativa de la gestión integrada de la salud y el ambiente" (IEGISA), en cuyo marco los docentes participantes diseñan y ejecutan, en sus centros educativos, acciones curriculares y extracurriculares en este campo, apoyados por especialistas de la zona. Los docentes participantes son convocados por las autoridades competentes del Ministerio de Educación Pública, de manera que están autorizados para dedicar tiempo laboral al desarrollo del curso. Además, reciben acreditación para el Servicio Civil por parte de las unidades académicas ejecutoras.

Este trabajo se acompaña con un eje paralelo de proyección comunitaria centrado en la incorporación de los padres de familia a los proyectos institucionales; estas actividades y las redes institucionales asociadas proveen a los docentes y centros educativos participantes la plataforma de proyección local deseada.

Se desarrolló un módulo de sistematización en el que se convocó a docentes seleccionados para que realizaran la sistematización en sus zonas. Se están desarrollando dos módulos nuevos: huertas, y monitoreo y restauración socioecológica (MRSE).

\section{Palabras clave}

Innovación didáctica, gestión ambiental, ambiente, educación primaria, educación secundaria, salud, biodiversidad, monitoreo.

\section{Abstract}

The objective of this project is to provide theoretical and practical tools for teachers with curricular or extracurricular activities of environmental management in primary, secondary or specialized education, so they can develop and evaluate proposals in this field. It seeks to create the conditions for public schools in the south, incorporating a component that stimulates the development of skills and abilities for public participation in environmental management at local areas. The project began with the Regional Office of Education at Perez Zeledón in 2012, the project was extended to the Regional Office of Education at Grande de Térraba (which includes the districts of Buenos Aires de Puntarenas and Osa).

The fundamental strategy of the project has focused on the development of a modular course Educational innovation of integrated health and the environment management (IE-GISA), under which participating teachers designed and implemented in their schools educational and extra curricular activities in this field, supported by specialists in the area. The participating teachers are called by the competent authorities of the Ministry of Education, so that they are authorized to spend time working at the course. They also receive accreditation for the Civil Service. This work is accompanied by a parallel axis of community outreach focused on incorporating parents in institutional projects, these activities and institutional networks provide a local projection.

We are developing three new modules: systematic, orchards and socio-ecological monitoring and restoration (MRSE).

\section{Key words}

Educational innovation, environmental management, environment, primary education, secondary education, health, biodiversity, monitoring. 


\section{Marco teórico}

La Constitución Política de Costa Rica en su artículo 50, según una reforma de 1994, establece que:

El Estado procurará el mayor bienestar a todos los habitantes del país, organizando y estimulando la producción y el más adecuado reparto de la riqueza. Toda persona tiene derecho a un ambiente sano y ecológicamente equilibrado. Por ello, está legitimada para denunciar los actos que infrinjan ese derecho y para reclamar la reparación del daño causado.

El Estado garantizará, defenderá y preservará ese derecho. La ley determinará las responsabilidades y las sanciones correspondientes.

El I de enero del 2005 arrancó la "Década de la Educación por la Sostenibilidad", promovida por la Naciones Unidas mediante la Resolución 57/254 de la Asamblea General, que declaró el periodo 2005-20I 4 como un decenio dedicado al propósito de fortalecer la educación y reorientarla hacia los objetivos del desarrollo sustentable.

La Ley Orgánica del Ambiente (1995) de Costa Rica, en su artículo 12, establece que el Estado, las municipalidades y las demás instituciones, públicas y privadas, fomentarán la inclusión permanente de la variable ambiental en los procesos educativos, formales y no formales, de los programas de todos los niveles. El objeto será adoptar una cultura ambiental para alcanzar el desarrollo sostenible. Además, en el artículo 13 se señala algunas características que debe tener la educación ambiental, tales como su capacidad de relacionar los problemas del ambiente con las preocupaciones locales y la política nacional de desarrollo; asimismo, debe incorporar el enfoque interdisciplinario y la cooperación como principales fórmulas de solución, destinadas a promover la conservación y el uso sostenible de los recursos naturales.

Por otra parte, la Ley de Biodiversidad (1998), en su artículo 86, señala que la educación biológica deberá ser integrada en los planes educativos en todos los niveles previstos, para lograr la comprensión del valor de la biodiversidad, el modo en que desempeña un papel en la vida y es la aspiración de cada ser humano. El Ministerio de Educación, en coordinación con las entidades públicas y privadas competentes en la materia, en especial el Ministerio del Ambiente y Energía, deberá diseñar políticas y programas de educación formal que integren el conocimiento de la importancia y el valor de la biodiversidad, así como el conocimiento asociado, las causas que la amenazan y reducen, y el uso sostenible de sus componentes, a fin de facilitar el aprendizaje y valoración de la biodiversidad que rodea a cada comunidad, y demostrar el potencial de ella para aumentar la calidad de vida de la población.

El Sistema Nacional de Áreas de Conservación (SINAC) debe fomentar y desarrollar programas de formación ambiental en todos los niveles educativos del sistema formal y no formal; en el caso del sistema formal, lo hace a solicitud del Ministerio de Educación Pública (MEP) como ente rector en esta materia y a través de la Oficina de Educación Ambiental del Ministerio de Ambiente y Energía (MINAE).

El enfoque educativo del SINAC promueve un concepto de extensión ambiental, con procesos de capacitación y organización comunitaria que toman como punto de partida los problemas y alternativas de solución existentes a escala local y regional, mediante una estrategia de coordinación intra e interinstitucional, que incluye los programas educativos del MEP. Con este fin, busca diseñar programas novedosos de educación ambiental con ayuda de alianzas estratégicas con organizaciones e instituciones calificadas en cada área de conservación, y en coordinación con el MEP, para la ambientalización curricular, sin dejar de lado las áreas silvestres protegidas como aulas abiertas y laboratorios vivientes.

El Plan deAcción de la Educación paraTodos 2003-20 I 5 , del Ministerio de Educación Pública (2002), incluye al programa "Ética, Estética y Ciudadanía" como uno de sus elementos estratégicos, cuyo objetivo es "promover la formación ética, estética y ciudadana del estudiantado mediante la incorporación de estas tres perspectivas en el Currículum Nacional Básico". Dicho objetivo está asociado, entre otras, a la siguiente meta:

5.3.- Fortalecimiento del eje transversal de valores en sus cuatro temas (educación integral de la sexualidad humana, educación para la salud, cultura ambiental para el desarrollo sostenible, vivencia de los derechos humanos para la democracia y la paz), mediante proyectos anuales que involucren en los centros educativos al profesorado, al estudiantado y a la comunidad educativa en general. 
Se busca la promoción del conocimiento, sensibilidad valores y competencias necesarias para inculcar un estilo de vida saludable en las poblaciones estudiantiles de todos los niveles y modalidades del sistema educativo, en el marco de una relación armoniosa con la naturaleza y una educación para el desarrollo sostenible.

Las acciones para conseguir el cumplimiento de esta meta están orientadas a fortalecer el desarrollo integral de la persona con ayuda de un currículo que incorpore la vivencia de valores y actitudes en el estudiante que le permitan el desarrollo de destrezas y habilidades para vivir plenamente en sociedad y en armonía con el ambiente, equilibrando el cúmulo de conocimientos recibidos en la institución educativa con su desarrollo pleno.

El Plan Nacional de Salud 2010-202I (Ministerio de Salud, 2010) establece como objetivo general $N^{\circ} 2$ contribuir al desarrollo sostenible del hábitat humano mediante la protección, restauración, mejoramiento y uso del ambiente, para promover una adecuada calidad de vida. A la vez, señala como objetivos específicos los siguientes:

- Impulsar acciones para el control, protección y restauración de la naturaleza, y los consecuentes efectos del cambio climático.

- Disminuir, de manera sostenible, los niveles de contaminación atmosférica, sónica, de suelos y de aguas que inciden sobre la calidad de vida de la población.

- Promover la seguridad alimentaria y nutricional mediante sistemas de producción, comercialización y consumo de alimentos propiciadores de la salud, bajo criterios de inocuidad y sostenibilidad socioambiental.

- Desarrollar una gestión intersectorial para la disposición, tratamiento y reutilización de los residuos sólidos, líquidos y gaseosos, con sostenibilidad ambiental, social y económica.

El XVII Informe del Estado de la Nación (Conare, 20II) incluye una primera indagación sobre el tema de ambiente y educación: su presencia en el sistema educativo formal y no formal, las iniciativas existentes a nivel local y las que desarrollan diversas organizaciones, y los desafíos de investigación en los que se debe profundizar en posteriores ediciones de este informe. Se señala que persisten brechas en materia de infraestructura y oferta educativa que afectan, de modo negativo, zonas rurales, centros públicos y ciertas direcciones regionales, principalmente aquellas que se ubican en zonas costeras y fronterizas.

ElTercer Informe del Estado de la Educación (Conare, 20II) establece que, en materia de formación y desarrollo profesional docente, los estudios más recientes y las mejores prácticas internacionales indican que la calidad de los maestros y profesores es el factor que más influye en la calidad de la educación. Para avanzar en este ámbito, es importante mejorar los procesos de selección de los estudiantes en las carreras de Educación y, en particular, lograr la acreditación de calidad de los programas universitarios de formación docente.

Una necesidad urgente es que el MEP cuente con el perfil del educador que desee contratar y que pida como requisito la acreditación de la carrera universitaria de la que provienen las personas interesadas. Por último, para los docentes en ejercicio, es fundamental que el MEP articule una oferta de desarrollo profesional continuo orientada por estándares y niveles de calidad esperados, a fin de superar el desorden imperante en esta materia.

La educación es un instrumento clave de la sociedad costarricense para promover el desarrollo humano, ya que potencia en las personas la adquisición de un conjunto de habilidades, destrezas y valores que les permiten desarrollar la autonomía, la creatividad y la razón práctica necesarias para desenvolverse como agentes morales (con habilidades cognitivas para detectar razonablemente las opciones que están a su disposición, decidir y asumir las consecuencias), como agentes políticos (con habilidades para desempeñarse como miembros activos de una comunidad política democrática) y como agentes sociales (con habilidades para actuar como integrantes de una sociedad y su cultura, capaces de convivir con los otros, respetar el medioambiente y aprovechar las oportunidades empresariales y de empleo).

Retener a los estudiantes a partir de una oferta académica atractiva sigue siendo un desafío, que pasa por una revisión profunda del currículo de primaria y secundaria, y las diversas modalidades de estudio, cuyos resultados hoy se desconocen. La estructura curricular en secundaria no cambia desde los años setenta y tiene notables desequilibrios 
entre contenidos y actividades, poca articulación entre ciclos y materias, y una aplicación homogénea y descontextualizada.

Todo esto constituye una barrera para el desarrollo de un aprendizaje más significativo en los alumnos. Las reformas a los programas de estudio impulsadas en el marco del programa "Ética, Estética y Ciudadanía", así como la promoción de una enseñanza de las ciencias basada en la indagación y el establecimiento de estándares para el uso de las tecnologías de información y comunicación, son esfuerzos positivos en esta dirección.

La salud humana no se puede considerar aisladamente, pues depende enormemente de la calidad del ambiente en que la gente vive: para que la gente esté sana se necesita un ambiente sano.

Los enfoques ecosistémicos en salud humana el enfoque de Ecosalud- son nada menos que la ubicación de la humanidad en el medio ambiente. El enfoque de Ecosalud reconoce que hay nexos inextricables entre los humanos y su ambiente biofísico, social y económico, que se reflejan en la salud del individuo.

El Enfoque Ecosistémico en Salud Humana estuvo relacionado con el desarrollo global de la ecología durante la segunda mitad del siglo $X X$. Al comienzo, la ecología adoptó una perspectiva basada principalmente en los aspectos biofísicos de un ecosistema. En efecto, todavía algunas personas consideran que la ecología es la manera de restaurar los ecosistemas a su estado primitivo. Frente a la realidad de una población mundial de unos 6300 millones de habitantes, que puede llegar a ser de 9000 o 10000 millones en los próximos 50 años, es muy difícil excluir a los humanos de la ecuación ecológica. Por eso ahora, y cada vez con más frecuencia, se incluye a las comunidades humanas en la descripción de los ecosistemas contemporáneos.

Para aquellos con una visión holística, la humanidad, con sus aspiraciones y su universo cultural, social y económico, está en el centro del ecosistema, con una posición igual a la de los parámetros biofísicos. Los elementos vivientes y no vivientes de la naturaleza interactúan con un equilibrio dinámico que, mejor manejado, debería asegurar el desarrollo sostenible de las comunidades humanas.

El enfoque de ecosalud es antropocéntrico: la gestión de los ecosistemas se plantea como la búsqueda del equilibrio óptimo de la salud y el bienestar del ser humano, no solo la protección del medio ambiente. Por lo tanto, su objetivo no es preservar el medio ambiente como era antes de que aparecieran los asentamientos humanos. La presencia de los seres humanos creó una nueva dinámica en la que deben considerarse las aspiraciones sociales y económicas de la gente, particularmente porque la gente tiene el poder del control, del desarrollo y del uso de su ambiente de manera sostenible, o de abusar de él. Este es un aspecto original de dicho enfoque.

Otro aspecto original es la adopción de un proceso de investigación que no está restringido a los científicos, de manera que los conocimientos adquiridos puedan integrarse a la vida de las personas. El desafío es satisfacer las necesidades humanas sin modificar o sin arriesgar el ecosistema a largo plazo, e idealmente mejorarlo.

Cuando los científicos de varias disciplinas les dan participación tanto a los individuos de la comunidad que estudian como a quienes toman las decisiones, se puede decir que operan dentro de un esquema transdisciplinario. Los científicos que usan el enfoque transdisciplinario envían una señal clara de que van a considerar los diferentes aspectos del problema, dando activa participación en su trabajo a la población local, así como a quienes toman las decisiones.

Si los problemas sociales se pueden enunciar en forma de interrogantes que puedan tratarse en un proceso científico, las comunidades podrán expresar lo que esperan de los científicos y de quienes toman las decisiones, y esto, a su vez, conduce a soluciones "socialmente fuertes"; estos son solo dos de los muchos beneficios potenciales del enfoque transdisciplinario.

Por tanto, la transdisciplinariedad implica que participen no solo los científicos, sino los representantes de la comunidad y otros actores que, fuera de disponer de un conocimiento particular del problema, tienen también un papel y un interés en su solución. Estos "no científicos" pertenecen con frecuencia a las ONG o a las agencias del gobierno. El enfoque transdisciplinario les da el derecho de ser oídos y, por tanto, de compartir sus experiencias, conocimientos y expectativas.

El enfoque transdisciplinario es muy distinto de la investigación unidisciplinaria, que caracteriza a las ciencias experimentales como la química o la física, 
y a las ciencias teóricas, como las matemáticas. También se diferencia del enfoque interdisciplinario, que estudia los fenómenos en la intersección de dos disciplinas usualmente cercanas la una de la otra, como es el caso de la bioquímica, originada en la superposición entre la biología y la química. Tampoco equivale a la multidisciplinariedad, en la cual los investigadores de diferentes disciplinas trabajan juntos con el fin de enriquecer su propia comprensión como resultado de los aportes de sus colegas, pero donde la coordinación no necesariamente conduce a acciones integradas (Lebel, 2005).

\section{Metodología}

\section{Coordinación con Direcciones Regionales de Educación}

El proyecto inicia con reuniones de coordinación con autoridades del MEP para definir los circuitos escolares y los centros educativos que participan en el proceso anual de formación de educadores. En los años 2010 y 2011 , esto se trabajó en coordinación con la Dirección Regional de Educación de Pérez Zeledón y Grande de Térraba. Para el 2012 se coordinó con dichas direcciones y se incluyó la Dirección Regional de Coto (cantones de Coto Brus, Golfito y Corredores).

Por último, se realizó una primera reunión con el director regional de cada zona para exponerle el alcance del proyecto y la importancia de la participación activa de las autoridades del MEP.

\section{Talleres de inducción}

Al inicio de cada año, se llevan a cabo talleres dirigidos a los supervisores, asesores y directores de los centros educativos participantes durante ese año, con el fin de dar a conocer los objetivos del proyecto y sus alcances. Se hace entrega de los materiales de trabajo a cada centro educativo incluido en el proyecto durante ese año.

\section{Selección de equipo de apoyo para las visitas a proyectos}

Se seleccionó un equipo de apoyo entre docentes que han participado en años anteriores en el proyecto. Con esto se busca ir formando al personal de las zonas involucradas de modo que se pueda transferir la ejecución del proyecto y su continuidad al MEP. Cada docente del equipo de apoyo entrega un informe mensual detallado de sus actividades.

\section{Módulo introductorio}

Por medio de la Dirección Regional correspondiente, se convoca a los profesores y maestros, que participarán del curso modular, al módulo introductorio, con una duración de una semana, en el cual se dan las bases metodológicas del curso y se introducen los conceptos que guiarán su desarrollo a lo largo del año. Al inicio de este módulo, se llevó a cabo una evaluación preliminar del grado de conocimiento y un recuento de las acciones que los docentes han ejecutado hasta este momento, antes de iniciar el curso modular. Esta información es la línea base, o punto de partida, que será comparada con el estado al finalizar el año.

Se realiza una vez al año, cada año con nuevos docentes. Este módulo introductorio de cuarenta horas presenciales, es una introducción para incorporar la temática ambiental y de salud en el currículo escolar, ejes básicos para integrar en las instituciones educativas la gestión ambiental. Los participantes concluyen este módulo con una propuesta de proyecto para ser desarrollada en la institución educativa en la cual laboran.

\section{Talleres con los educadores}

El curso modular consta de cinco módulos de cuarenta horas cada uno; se construye a lo largo de un año, con el propósito de impulsar y acompañar esta construcción de la transversalidad, con una inducción conceptual y, a la vez, práctica en la temática de la gestión integrada de la salud y el ambiente.

El módulo introductorio, con la propuesta de proyecto que desarrolla el grupo de docentes de cada centro educativo, y los cuatro talleres incluyen los módulos que componen el curso en el 2010. En el 20 I I se introdujo el módulo de sistematización y en el 2012 se introdujeron los módulos de huertas y de monitoreo. El curso-taller de sistematización duró un semestre (setenta y dos horas certificadas), y fue organizado conjuntamente con el Instituto de Investigación en Educación (INIE) de la Universidad de Costa Rica.

De los cuatro talleres que se realizaron anualmente en el 2010 y $20 \mathrm{II}$, dos se desarrollaron como giras 
de campo a empresas u organizaciones que tienen proyectos ambientales en las zonas aledañas a los centros educativos. Los talleres brindan elementos teóricos y prácticos para reforzar el conocimiento de los docentes y para apoyar el desarrollo de los proyectos que cada uno está ejecutando en su centro educativo. Los talleres duran un día y se celebra uno por mes. Los docentes asisten con el respaldo de una convocatoria emitida por las autoridades de la Dirección Regional.

\section{Visitas a los proyectos de los centros educativos}

El equipo interuniversitario y los docentes del equipo de apoyo realizan visitas, a lo largo del año, a los centros educativos con el fin de dar seguimiento y apoyar el desarrollo de los proyectos que están ejecutando los docentes. En cada visita se aplica una evaluación a cada docente del avance del proyecto y de la mejora en la oferta educativa que se brinda a los estudiantes.

\section{Cierre del curso modular}

Se realiza una sesión para presentar los proyectos desarrollados por cada docente o grupo de docentes en los centros educativos. Tiene una duración de un día. En esta sesión evalúan los logros obtenidos con la participación de los docentes en el proyecto. Se hace entrega de los títulos correspondientes a los participantes.

\section{Reuniones de coordinación del equipo interuniversitario}

Se realizaron reuniones de coordinación, evaluación de avances y propuesta de mejoras del proyecto a lo largo de cada año.

\section{Encuentro nacional de experiencias exitosas}

Al final del año, se organizó un encuentro en el que se seleccionaron los mejores proyectos de cada zona donde opera el proyecto. Cada docente participante en el Encuentro hizo una exposición oral de su proyecto. También se presentan los resultados de la sistematización de la experiencia.

\section{Módulo de sistematización}

Se seleccionaron docentes que han participado en años anteriores, o bien del año en curso, para que recibieran una capacitación sobre la sistematización de experiencias; se les dio seguimiento en el proceso y se les pidió la entrega de un producto concreto del módulo, que podía ser un video, un artículo o cualquier producto que permitiera dar a conocer la experiencia generada mediante la participación en el proyecto.

\section{Módulo de huertas orgánicas, y} monitoreo y restauración socioecológica del territorio - MRSET

Estos módulos se están desarrollando para el 2012. Se busca desarrollar la producción orgánica en los centros educativos, al igual que el monitoreo de ríos y de flora y fauna, como complemento al desarrollo del curso modular. El módulo de huertas se realiza en coordinación con el Programa Nacional de Huertas del MEP.

El módulo de monitoreo y restauración socioecológica (MRSE) se ha diseñado mediante la aplicación del enfoque del proyecto internacional GLOBE de educación en ciencias, que impulsa el Programa Nacional de Informática Educativa (Pronie) del MEP. Se usará un equipo especializado en monitoreo y un componente de "laboratorio virtual" impulsado por la UNED, en una plataforma de información geográfica. El propósito del módulo MRSE es que docentes y estudiantes, así como otros usuarios comunitarios, trabajen con datos locales en los laboratorios de informática de sus centros educativos y centros comunitarios inteligentes (CECI), monitoreando los temas de salud y ambiente, y elaborando, sobre esta base de información geográfica, propuestas de transformación en este campo.

\section{Organización y ejecución de talleres semestrales de coordinación con contrapartes regionales}

Se realizan reuniones de coordinación entre universidades, Direcciones Regionales de Educación del MEP, los Ministerios de Salud y Ambiente, organizaciones ambientalistas, empresas de la zona, ONG y otros, con el fin de ir fortaleciendo las redes de apoyo para los centros educativos y sus proyectos.

\section{Sitio web del proyecto}

Se inició el sitio, el cual se fortalece con la información que se genera a lo largo del desarrollo del proyecto. 


\section{Resultados y discusión}

\section{Coordinación con Direcciones Regionales de Educación}

Las reuniones de coordinación con autoridades del MEP para definir los circuitos escolares y los centros educativos que participarían en el proceso anual de formación de educadores durante el 2010, 201। y 2012 fueron exitosas. Se contó con el apoyo de la Dirección Regional de Educación de Pérez Zeledón y de la Dirección Regional de Grande de Térraba.

Las autoridades del MEP se comprometieron a realizar las convocatorias correspondientes de los docentes para cada una de las actividades del proyecto. Al cumplirse a cabalidad este compromiso, se garantizó el éxito de las actividades programadas. Es fundamental el apoyo del MEP, pues demuestra su interés en el desarrollo de este tipo de proyectos. Esto es un avance importante en el camino hacia la transferencia del proyecto al MEP, de modo que el su desarrollo no dependa de la presencia de las universidades, aun cuando el apoyo universitario se mantenga en el futuro.

\section{Talleres de inducción}

El apoyo recibido de los supervisores y asesores de los centros educativos en el 2010 no fue total. Las otras labores asignadas les impidieron apoyar más el proyecto. Para el 20I I esta situación mejoró y aún más en el 2012, con un compromiso de participación de asesores y supervisores en visitas colegiadas y otras acciones de seguimiento.

\section{Selección de equipo de apoyo para las visitas a proyectos}

Para el 2010 se contrató personal de la zona, pero para el 20 I I se seleccionaron cuatro docentes para que conformaran el equipo de apoyo al proyecto. Estos docentes ya habían llevado el curso modular y fueron contratados para dar seguimiento a los proyectos de los docentes que estaban desarrollando uno durante este año.

En octubre del 2010, se organizó un curso de Formación de formadores, con el fin de fortalecer la preparación de los profesores que habían participado en el grupo de apoyo y que apoyarían el proceso en los siguientes años. Cuatro de los participantes participaron activamente en el desarrollo del módulo introductorio del grupo del $201 \mathrm{l}$.
El objetivo de formar a estos docentes en la metodología del curso modular es contar con personal de las zonas de trabajo que apoye la continuidad del proyecto, conforme se transfiere al MEP, y garantizar, de esta forma, que si el papel de las universidades dentro del proyecto cambia, se continuará con él. Dichas acciones están encaminadas a garantizar la continuidad del proyecto, aún en el caso de que las universidades pasen a ser solamente un acompañante en el proceso.

\section{Módulo introductorio}

Por medio de las direcciones regionales correspondientes, se convocó a los profesores y maestros que participaron en el módulo introductorio. No todos los docentes que participan en este curso terminan el proceso.

\section{Talleres con los educadores}

A lo largo del 2010, se logró el desarrollo de los cuatro talleres y dos de ellos se desarrollaron en la modalidad de giras de campo. Se recibió el apoyo de las direcciones regionales para la convocatoria de los docentes.

Cada taller responde a los módulos de planeamiento didáctico, de gestión de la contaminación y de gestión de la biodiversidad. Es decir, los módulos se desarrollaron mediante talleres de un día con temas didácticos y ambientales que pueden ser incorporados en las lecciones diarias.

Las giras permiten que la experiencia adquirida por habitantes de la zona pueda emplearse como insumo para los educadores que participan. De esta forma se sensibiliza a los docentes en la problemática de salud y ambiente que afecta a su institución o al entorno inmediato, con lo que se mejora la capacidad de construir opiniones y se puede crear conciencia en los niños sobre la problemática.

Las giras permitieron a los docentes reforzar el desarrollo de sus proyectos en los centros educativos. Como ejemplo de este efecto, se señala el hecho de que, luego de visitar fincas con manejo orgánico, los docentes decidieron continuar sus proyectos con este tipo de manejo y algunos ampliaron su proyecto e incluyeron la producción de hortalizas orgánicas.

En el 201 I se inició el proceso para desarrollar el curso en forma bimodal (presencial y no presencial). 


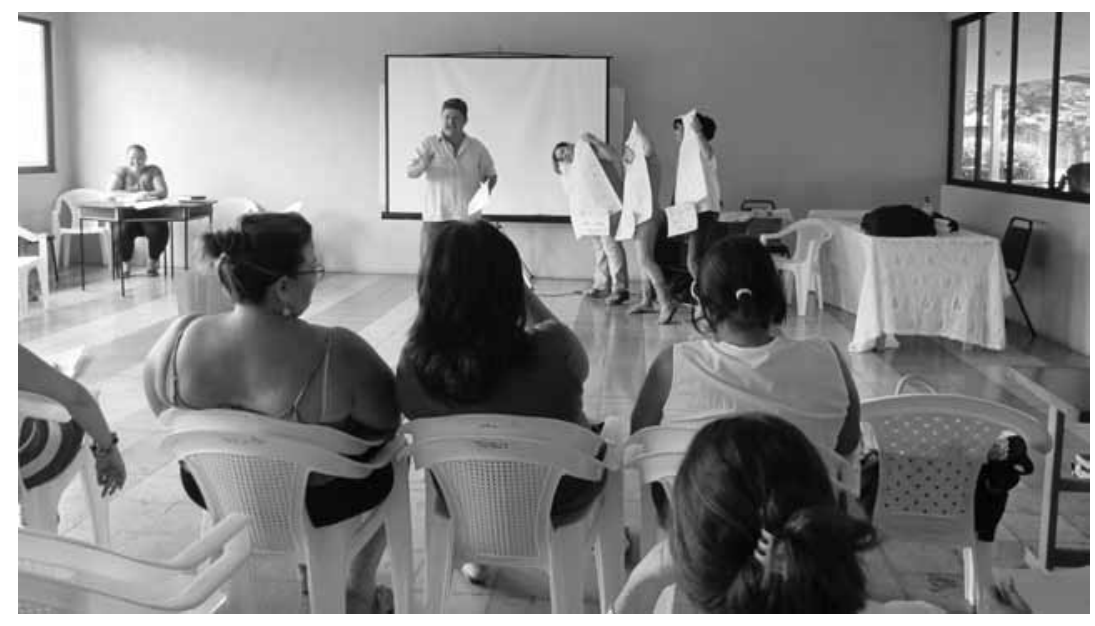

Figura I.Taller en Pérez Zeledón, 2010.

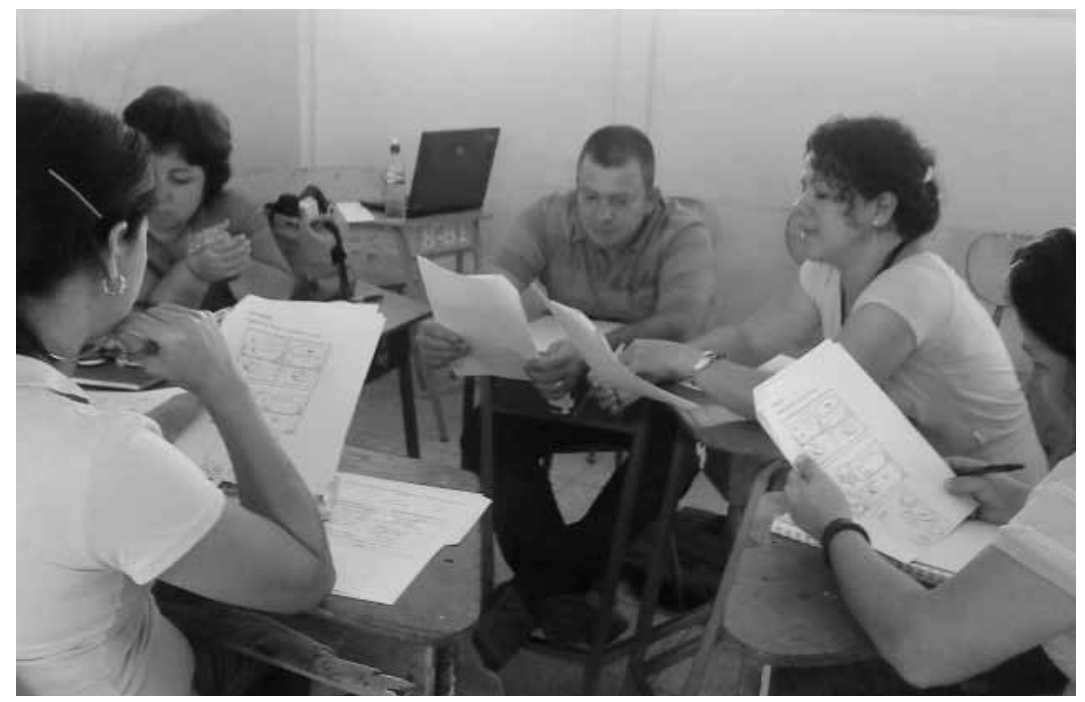

Figura 2. Taller realizado en el Colegio Yolanda Oreamuno, $201 \mathrm{I}$.

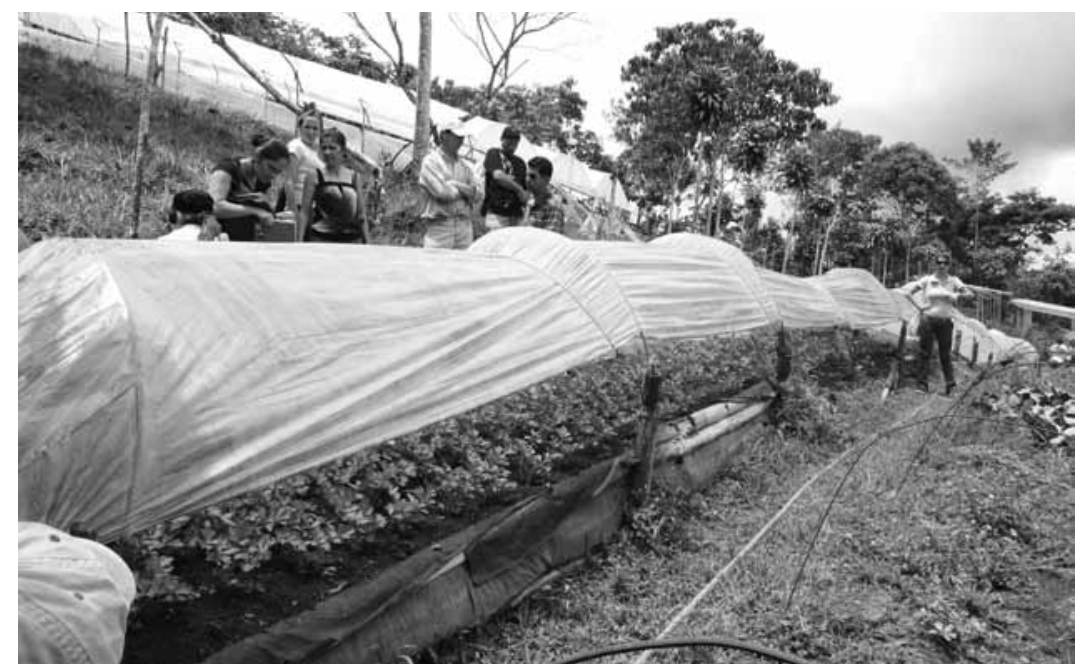

Figura 3. Gira a finca orgánica, 2010. 


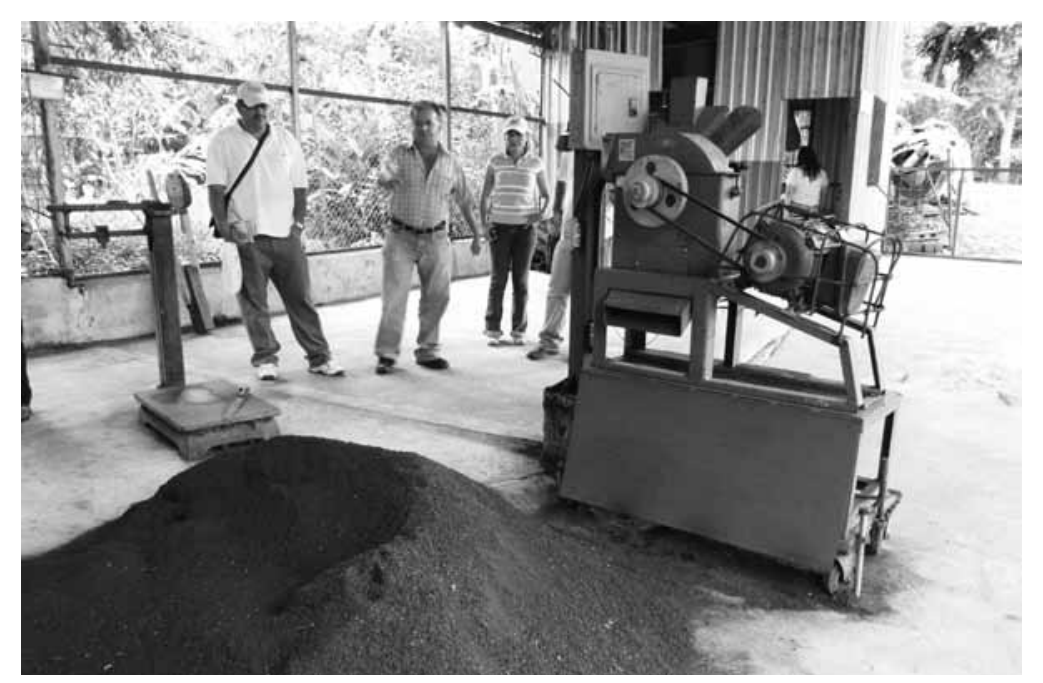

Figura 4.Visita a empresa que procesa sólidos de aguas negras.

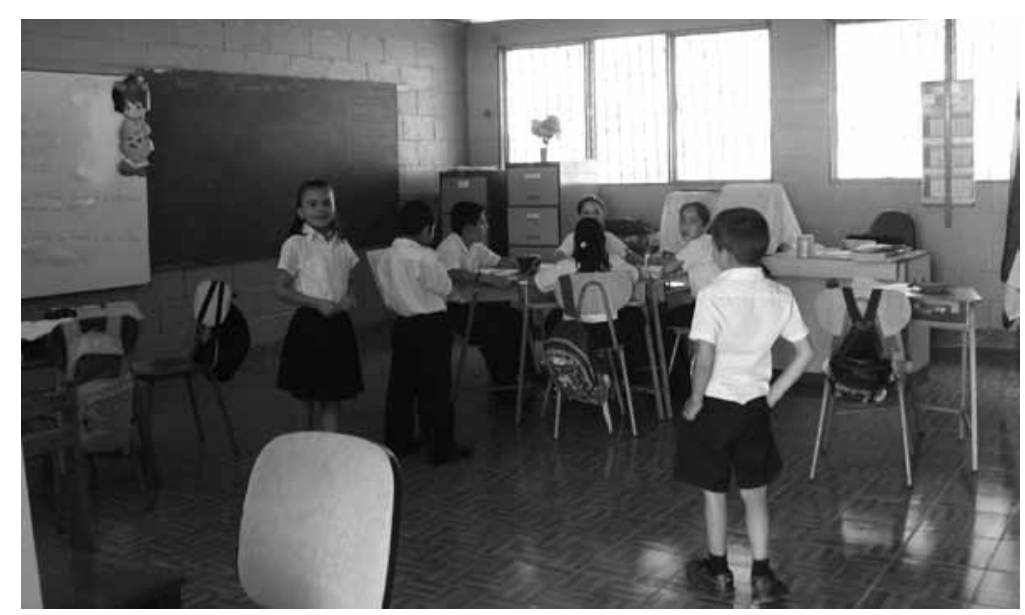

Figura 5.Visita a Escuela El Alto de la Trinidad, 2010.

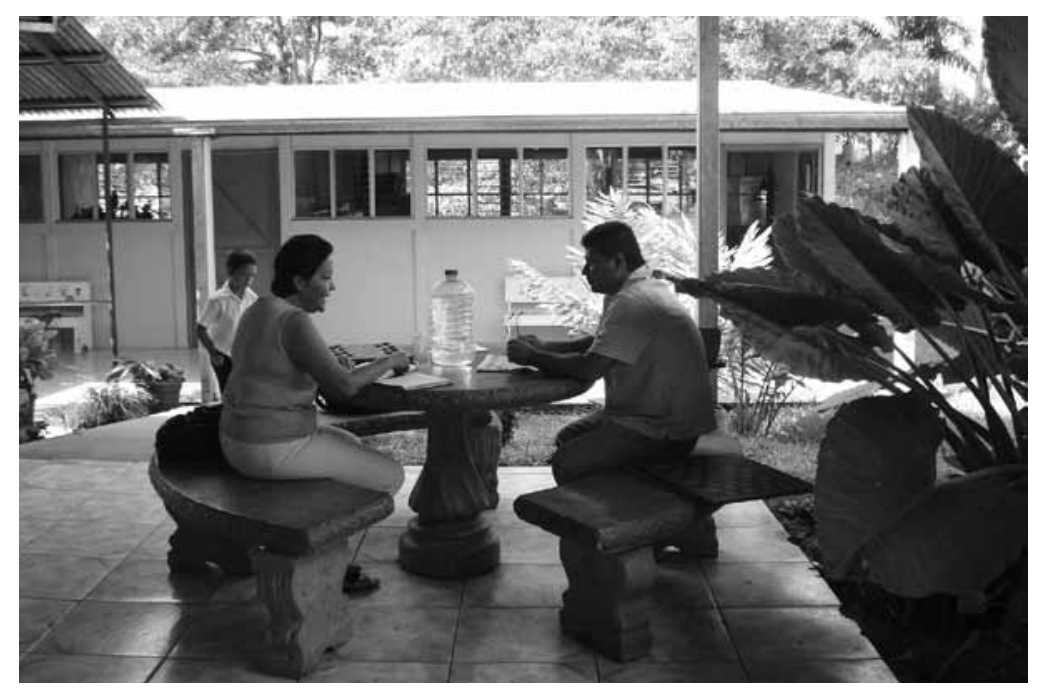

Figura 6.Visita a Escuela Tinamastes. 


\section{Visitas a los proyectos de los centros educativos}

Durante el 20 I 0 y el 20 I I, el equipo interuniversitario y los docentes del equipo de apoyo realizaron visitas a los centros educativos con el fin de dar seguimiento y apoyar el desarrollo de los proyectos que estaban ejecutando los docentes. En cada visita se aplicó la evaluación a cada docente del avance del proyecto y de la mejora en la oferta educativa que se brinda a los estudiantes. Se observó cómo los educadores lograron aplicar las estrategias didácticas trabajadas en los talleres con sus estudiantes en el aula.

\section{Cierre del curso modular}

Se realizaron sesiones de presentación de los proyectos desarrollados por cada docente o grupo de docentes en los centros educativos en el mes de octubre del 2010 y 2011 . Se evaluaron los logros obtenidos con la participación de los docentes en el proyecto. Posteriormente, se hizo entrega de los títulos correspondientes a los participantes.

La lista de instituciones, docentes y proyectos desarrollados en el 2010 se detalla en el siguiente cuadro:

\begin{tabular}{|c|c|c|c|}
\hline Institución educativa & Circ. & Participante & Proyecto \\
\hline \multicolumn{4}{|c|}{ Dirección regional de educación de Grande de Térraba } \\
\hline $\begin{array}{l}\text { I. Liceo } \\
\text { Oreamuno }\end{array}$ & $\begin{array}{c}\text { Buenos } \\
\text { Aires } \\
01\end{array}$ & \begin{tabular}{|ll} 
1. & Anais Vargas \\
2. & Ronald Saborío \\
3. & Adriana López \\
4. & Dixia Rivera \\
5. & Mónica Barrantes \\
\end{tabular} & $\begin{array}{l}\text { Manejo adecuado de los desechos sólidos en el colegio. } \\
\text { Monitoreo biológico en las aguas del Río Volcán. }\end{array}$ \\
\hline \multicolumn{4}{|c|}{ Dirección regional de educación de Pérez Zeledón } \\
\hline 2. Esc. Barrio Nuevo & 08 & 6. Hellen Cerdas & Mejoramiento del jardín escolar. \\
\hline 3. El Alto de la Trinidad & 08 & 7. Lorena Méndez Umaña & Huerta hidropónica. \\
\hline 4. Esc. Nueva Santa Ana & 06 & 8. Lorena Navarro Fallas & $\begin{array}{l}\text { Cambio de actitud ante el problema de los desechos } \\
\text { sólidos. }\end{array}$ \\
\hline 5. Esc. Daniel Flores Z. & 05 & \begin{tabular}{|ll} 
9. & Carolina Quesada \\
10. & Loreli Jara \\
I I. & Rita Varela \\
\end{tabular} & $\begin{array}{l}\text { Desarrollo de una huerta orgánica, manejo de los } \\
\text { desechos sólidos, creación de un jardín de plantas } \\
\text { medicinales y reforestación de la quebrada. }\end{array}$ \\
\hline 6. Esc. Montecarlo & 06 & 12. Elena Araya Leiva & $\begin{array}{l}\text { Manejo adecuado de los desechos sólidos y la } \\
\text { importancia de reforestar. }\end{array}$ \\
\hline 7. Esc. La Unión & 06 & 13. Walter Solano Rojas & $\begin{array}{l}\text { Manejo adecuado de los desechos sólidos y la } \\
\text { reforestación de zonas verdes. }\end{array}$ \\
\hline 8. Esc. La Hermosa & 05 & 14. Celia Montoya Espinoza & $\begin{array}{l}\text { Vivero de árboles nativos de la zona y hospederos de } \\
\text { aves, para reforestar las márgenes del Río General. }\end{array}$ \\
\hline 9. Esc. IDA-Jorón & 03 & $\begin{array}{l}\text { 15. Marlen Mora } \\
\text { 16. Dirsee Díaz } \\
\text { 17. Lilliana Barrantes }\end{array}$ & $\begin{array}{l}\text { Manejo de los residuos sólidos y embellecimiento de las } \\
\text { zonas verdes. }\end{array}$ \\
\hline 10. Esc. La Piedra & 05 & 18. Freddy Machado Arias & Mejoramiento del invernadero orgánico. \\
\hline II. Esc. ElTirrá & 05 & 19. Gerardo Blanco Mena & Huerta escolar orgánica. \\
\hline 12. Esc. Herradura & 05 & 20. Cinthya Valverde Alvarado & $\begin{array}{l}\text { Huerta orgánica y mejoramiento de las zonas verdes de } \\
\text { la escuela. }\end{array}$ \\
\hline 13. Escuela Guadalupe & 05 & 21. Walter Monge Valverde & Aprovechamiento integral del medio escolar. \\
\hline 14. Esc. Canaán & 05 & 22. Yorleny Corrales Méndez & "Si la naturaleza muere, morimos nosotros". \\
\hline 15. Esc. Santa María & 06 & 23. Leonel Jiménez Rojas & Huerta orgánica, jardines y reciclaje. \\
\hline 16. Esc. Santa Elena & 05 & 24. Cecilia Céspedes Barrantes & La huerta orgánica escolar. \\
\hline 17. Esc. La Repunta & 07 & 25. Sidney Mª Salazar Vega & "Aprendiendo a ser parte del ambiente". \\
\hline 18. Esc. La Guaria & 04 & 26. Alexander Rojas Fernández & "El jardín terapéutico". \\
\hline 19. Esc. La Sierra & 07 & 27. Gustavo A. Benavides Garro & $\begin{array}{l}\text { Manejo adecuado de residuos sólidos orgánicos para } \\
\text { abono y su utilización en huertas y jardines. }\end{array}$ \\
\hline 20. Escuela Villa Ligia & 03 & 28. Eida Segura & "Queremos una institución más limpia". \\
\hline 21. Esc.Tinamastes & 04 & 29. Marvin Céspedes & Mejoramiento de los jardines escolares. \\
\hline 22. Liceo San Pedro & & 30. Sandra Quesada Quesada & Reciclaje de botellas y latas. \\
\hline 23. Liceo Las Mercedes & 06 & 31. Ivannia León & "Un jardín para mi colegio" \\
\hline 24. Escuela Melico Salazar & & 32. Carmen Cubero & Manejo de los desechos sólidos. \\
\hline 25. Esc. Sagrada Familia & 01 & 33. Gabriela Castro Bonilla & $\begin{array}{l}\text { "Manejemos adecuadamente los residuos sólidos de la } \\
\text { escuela" y huerta hidropónica. }\end{array}$ \\
\hline
\end{tabular}


La lista de instituciones, docentes y proyectos desarrollados en el 20 I I se detalla en el siguiente cuadro:

\begin{tabular}{|c|c|c|c|}
\hline Centro educativo & Circ. & Educador participante & Proyectos \\
\hline \multicolumn{4}{|c|}{ Dirección regional de educación de Pérez Zeledón } \\
\hline I. Escuela San Miguel & 08 & I. $\quad$ Arguedas Godínez Melvin & $\begin{array}{l}\text { "Buenas prácticas de alimentación igual a buena } \\
\text { calidad de vida"y "Protejamos el ambiente". }\end{array}$ \\
\hline 2. Escuela San Gabriel & 08 & Camacho Valverde Onix & Huerta orgánica y siembra de árboles frutales. \\
\hline Escuela Las Delicias & 08 & Castro Picado Rudy & Reciclaje y huerta escolar orgánica. \\
\hline 4. Escuela Las Mesas & 08 & 4. García Lobo Grace & Huerta escolar y separación de desechos sólidos. \\
\hline 5. Escuela El Águila & 08 & $\begin{array}{l}\text { 5. Jiménez García Marisella } \\
\text { 6. Mora Vega Roirán }\end{array}$ & "Ambientemos nuestra comunidad". \\
\hline 6. Escuela San Martín & 08 & 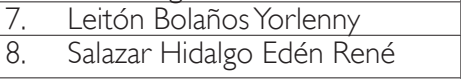 & $\begin{array}{l}\text { Huerta orgánica, talleres con materiales de desecho } \\
\text { y recolecta de materiales de desecho. }\end{array}$ \\
\hline 7. Escuela Santa Cecilia & 08 & 9. Martínez Cerdas Wendier & Huerta orgánica y reciclaje. \\
\hline 8. $\quad$ Escuela Bella Vista & 08 & 10. Monge Mora Jorge & $\begin{array}{l}\text { "Huerta saludable y mejora en la belleza escénica del } \\
\text { lote escolar". } \\
\text { "Reciclemos juntos". }\end{array}$ \\
\hline 9. Esc. San Antonio Abajo & 08 & 11. Rodríguez Jara Alicia & Huerta orgánica. \\
\hline 10. Escuela Valle La Cruz & 08 & 12. Solís Hodson Mayela & Huerta orgánica y arreglo de jardineras. \\
\hline I I. Escuela Santa Lucía & 08 & 13. Valverde Jiménez Silvana & Manejo de residuos sólidos y huerta orgánica. \\
\hline 12. Escuela Chimirol & 05 & $\begin{array}{|ll|}\text { 14. } & \text { Castro Méndez Iside } \\
\text { 15. } & \text { Benavides Garro Carolina } \\
\end{array}$ & "Reciclando para educar". \\
\hline 13. Escuela Daniel Flores & 05 & \begin{tabular}{|ll} 
16. & Rojas Ureña Maritza \\
17. & Navarro Arias Irma \\
18. & Vargas Rojas Jonathan \\
19. & Valverde Mora Jane \\
\end{tabular} & $\begin{array}{l}\text { Producción de hortalizas en dos sistemas, orgánico e } \\
\text { hidropónico, en un ambiente protegido. }\end{array}$ \\
\hline 14. CTP General Viejo & 05 & 20. Barrantes Núñez Caleb & "Porque yo sí quiero un cambio". \\
\hline 15. Escuela Pedro Pérez & 01 & \begin{tabular}{|ll} 
21. & Cubero Ureña Pamela \\
22. & Segura Godoy Ma. Leonor \\
23. & Mejía Torres Heidy \\
24. & Picado Castro Cinthya \\
25. & Acuña Arias Heilyn \\
\end{tabular} & Educación de la mano con el ambiente. \\
\hline 16. Escuela Sagrada Familia & 01 & \begin{tabular}{|ll} 
26. & Godínez Calvo Yancy \\
27. & Marín Mena Viviana \\
28. & Porras Castro Carla \\
29. & Sancho Maroto Roxana \\
30. & Muñoz Navarro Nury \\
\end{tabular} & $\begin{array}{l}\text { Manejo de residuos sólidos y embellecimiento de las } \\
\text { zonas verdes. }\end{array}$ \\
\hline $\begin{array}{l}\text { 17. Dirección Regional P.Z. } \\
\text { Asesoría pedagógica Asesor } \\
\text { Ciencias }\end{array}$ & & 31. Herrera Mora Rachid & $\begin{array}{l}\text { Colaboración con el proyecto de reciclaje de papel } \\
\text { en la Dirección Regional de P.Z. }\end{array}$ \\
\hline \multicolumn{4}{|c|}{ Dirección regional de educación Grande de Térraba } \\
\hline \multirow{4}{*}{$\begin{array}{l}\text { 18. Escuela Líder Rogelio } \\
\text { Fernández Guell }\end{array}$} & 01 & 32. Muñoz Umaña Rose Mary & \multirow{4}{*}{$\begin{array}{l}\text { "Guardianes del ambiente". } \\
\text { Embellecimiento de jardineras. } \\
\text { Huerta orgánica. }\end{array}$} \\
\hline & & 33. López Ortiz Ana Isabel & \\
\hline & & 34. Batista Méndez Annia Marieth & \\
\hline & & 35. Céspedes Azofeifa Ana Lorena & \\
\hline \multirow[t]{2}{*}{ 19. CTP de Buenos Aires } & 01 & 36. Herrera Arias Rolando & \multirow[t]{2}{*}{ Manejo de residuos sólidos. } \\
\hline & & 37. Jiménez Chamorro Yalena & \\
\hline 20. Escuela Santa Cruz & 01 & 38. Esquivel Urbina Albán & Huerta orgánica. \\
\hline
\end{tabular}

Fuente: Consejo Nacional de Rectores, 20I I.

\section{Encuentro nacional de experiencias exitosas}

En el 2010 se realizó el Encuentro en Sarapiquí, el 29 y 30 de noviembre. Los cuatro docentes seleccionados de la zona de Pérez Zeledón expusieron su proyecto en dicha actividad.

El Segundo Encuentro de Experiencias Exitosas MOE-GAUR se realizó en Dominical, el 3 y 4 de noviembre del 201 I. Ahí se presentaron también las sistematizaciones de los docentes que participan en el Primer Curso de Sistematización.

\section{Módulo de sistematización}

En el 201I se realizó el primer curso de sistematización, que inició el 29 de marzo y se 
completó el 15 de octubre con setenta y dos horas de duración, que incluía cinco sesiones mensuales de carácter presencial y trabajo no presencial. Participaron catorce docentes y asesores regionales, de los cuales cuatro presentaron su sistematización final en el Segundo Encuentro Nacional de Experiencias Exitosas MOE-GAUR.

Módulo de huertas orgánicas, y monitoreo y restauración socioecológica del territorio - MRSET

En el 20ll, se inició con acciones que apoyan el desarrollo del módulo de monitoreo. Se realizaron dos monitoreos biológicos y químicos en los ríos Sonador (Longo Mai de Volcán de Buenos Aires de Puntarenas) y Peñas Blancas, y en la quebrada Chumpulún (Pérez Zeledón) como inicio de la red de monitoreo que se pretende formar en el 2012. Esta red de monitoreo permitirá comunicar entre ellos la información del estado de contaminación del agua, además de compartir las actividades planeadas para la realización del monitoreo y, a su vez, enlazarse con centros de otras regiones y compartir la información para crear conciencia sobre el estado de los ríos en ciertas regiones del país.
Para el 20 I2, se diseñará el módulo para el pilotaje de redes interinstitucionales con los centros educativos participantes en 2011 y los seleccionados para el curso del 2012, a fin de que se realice el monitoreo de aguas, fauna y flora en sus comunidades.

Se tiene el perfil del módulo de huertas para ejecutarlo durante el 2012 .

\section{Organización y ejecución de talleres semestrales de coordinación con contrapartes regionales}

En el 2010, el apoyo de las instituciones no fue muy fuerte, por lo que se concluye que es necesario reforzar la participación del MEP.

En febrero del 2011 se realizaron visitas de coordinación a las instituciones y se realizó un taller de coordinación, en el que se contó con la participación de especialistas del MAG, de la Municipalidad, Ministerio de Salud, el MINAE y del Colegio de Ingenieros Agrónomos, EducaPILA. La coordinación mejoró considerablemente para este año, tanto en Pérez Zeledón como en Buenos Aires. Los especialistas de las instituciones han apoyado, con talleres y asesorías, a los educadores en el desarrollo de sus proyectos institucionales.

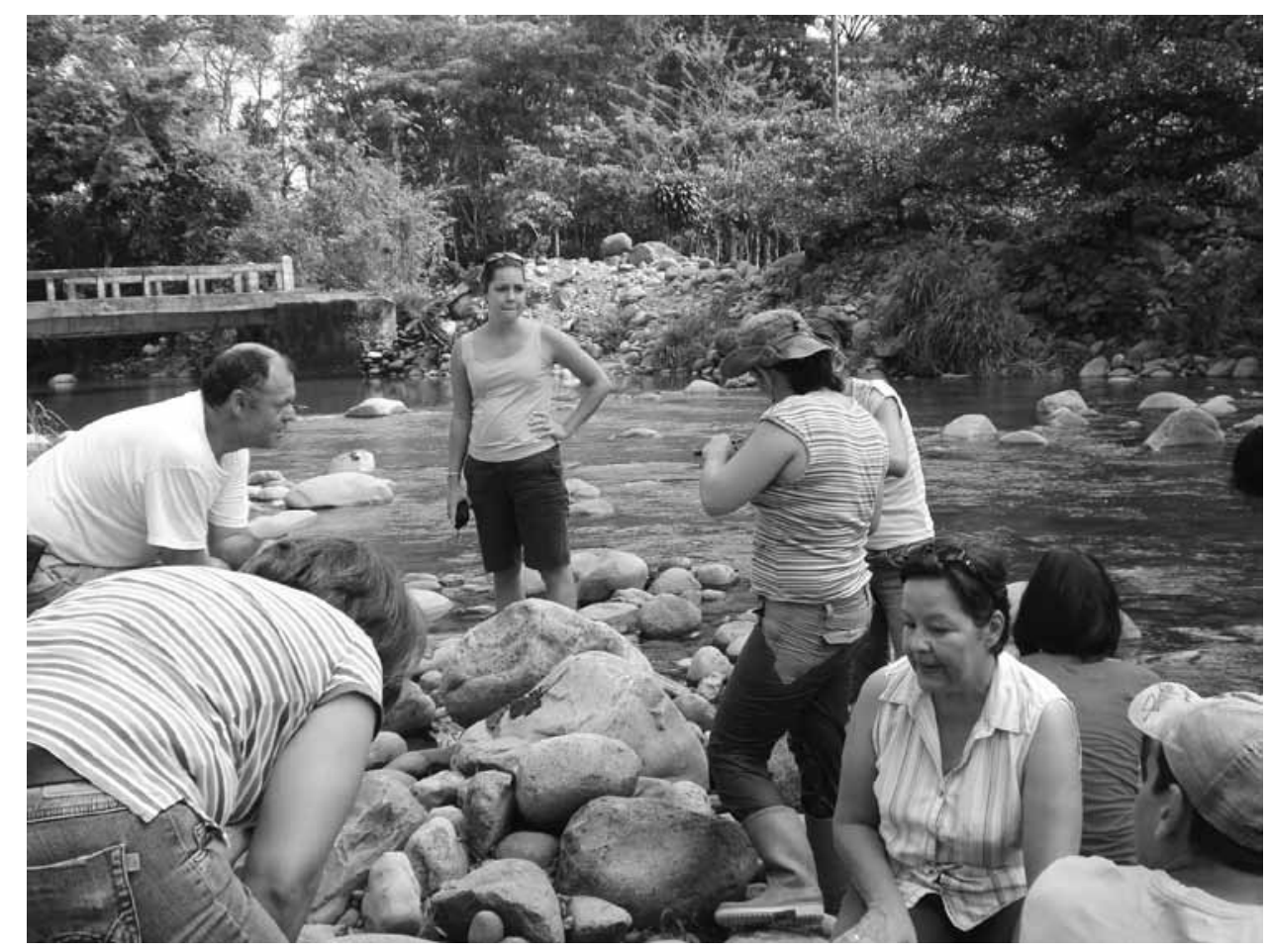

Figura 7. Monitoreo de río en Volcán. 


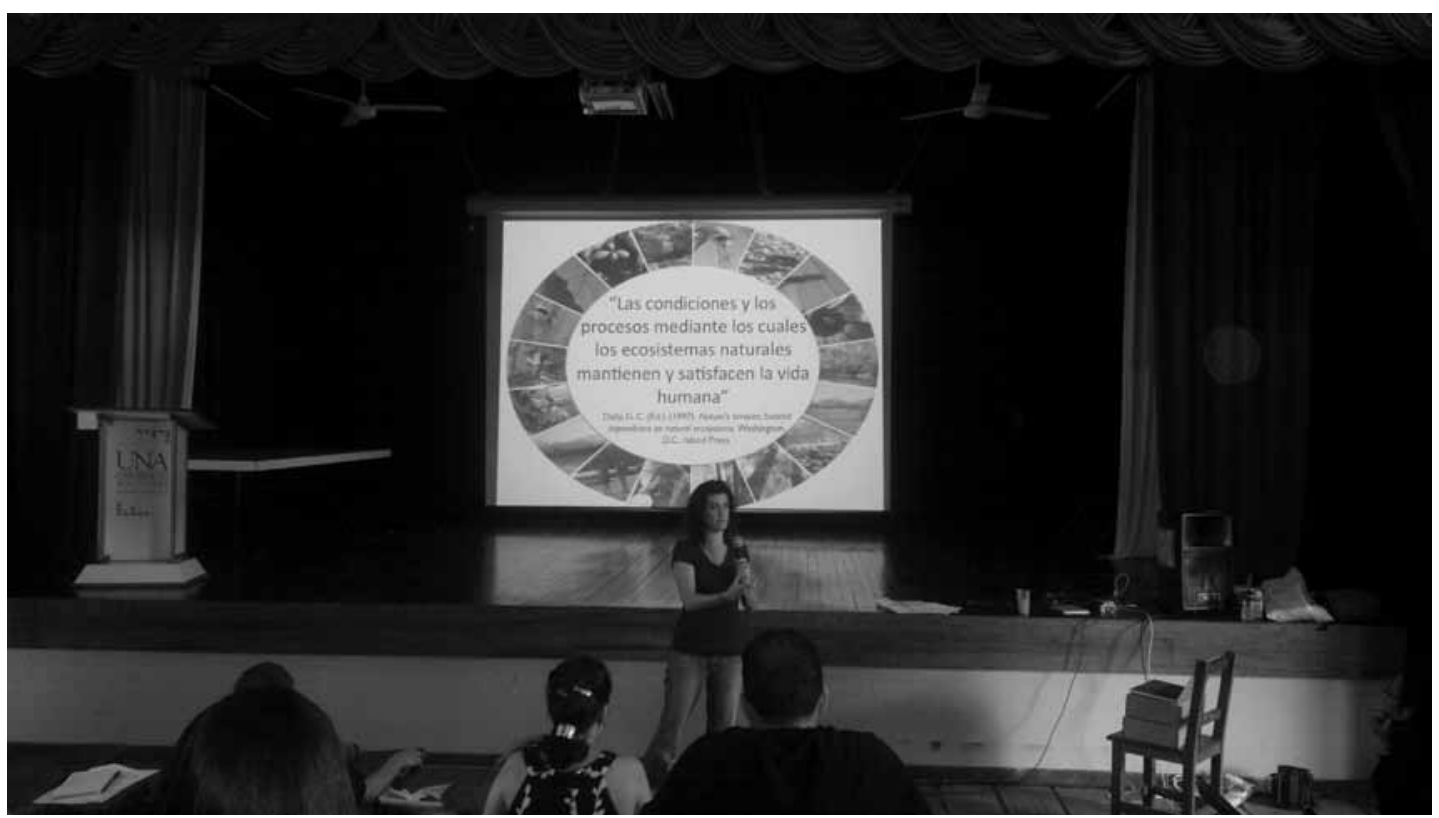

Figura 8. Taller de Ecosalud, 20 I I.

En ese mismo año se presentaron los resultados del proyecto en el primer y segundo foro semestral del Consejo de Participación Comunal, en la Dirección Regional Educativa de Pérez Zeledón y Grande de Térraba (Buenos Aires de Puntarenas).

\section{Sitio web del proyecto}

En el 20l I se inició la construcción del sitio y está en línea la primera versión: http://mgau.odd.ucr.ac.cr.

\section{Conclusiones}

El proyecto intenta construir la transversalidad en reversa, a partir de la ejecución de proyectos en los centros educativos donde los docentes participantes se encuentran colaborando cada uno desde su propia área disciplinaria.

Sehalogrado brindarherramientas teóricasy prácticas para que los docentes participantes desarrollen actividades curriculares o extracurriculares que incorporen el tema de la gestión ambiental en primaria y secundaria.

Los proyectos desarrollados en cada centro educativo participante son innovaciones didácticas que pueden contribuir a la formación de conciencia ambiental en los estudiantes, en los docentes y en las demás personas involucradas.
Se están formando redes interinstitucionales para monitoreo y restauración socioecológica, con perspectiva de cuencas, caudal ambiental y corredores biológicos. Hay dos experiencias iniciales sobre las que se busca trabajar como punto de partida: en Quizarrá de Pérez Zeledón y en Volcán de Buenos Aires de Puntarenas, donde hay antecedentes importantes de monitoreo de ríos, biodiversidad y situación socioeconómica.

En la Región Brunca ya hay siete docentes multiplicadores formados (en los cantones de Pérez Zeledón, Buenos Aires, Golfito y Coto Brus), cuya función en el marco de la plataforma bimodal propuesta para los años 20I 2-20I3 será -junto con los equipos académicos interuniversitarios- la de facilitadores en los componentes presenciales del curso IE-GISA y de "tutores" (en el sentido que da a este término la UNED) en los componentes no presenciales.

La sistematización de experiencias es crucial para el desarrollo futuro del proyecto, pues permite a los participantes enseñar y aprender a partir de las experiencias desarrolladas.

Se ha logrado un nivel de compromiso con el sector educación y un valioso enlace con los especialistas de la zona, que están dando apoyo y asesoría, tanto 
en los talleres como directamente a los educadores en la institución educativa.

Un ejemplo de la posición que el proyecto ha logrado en la zona de acción es el hecho de que sus principales actividades quedaron incorporados en el Plan Operativo Anual de la Dirección Regional Educativa de Pérez Zeledón, además de su presentación en los foros del Consejo de Participación Comunal realizados por las direcciones regionales de educación de Pérez Zeledón y Grande de Térraba.

\section{Bibliografía}

Consejo Nacional de Rectores. (201 I). Informe anual 2010 del Proyecto "Mejora de la oferta educativa en gestión ambiental urbana y rural: Experiencia piloto interuniversitaria en Pérez Zeledón". (p.28). San José. .

Consejo Nacional de Rectores. (2012). Informe anual 2011 del Proyecto "Mejora de la oferta educativa en gestión ambiental urbana y rural: Experiencia piloto interuniversitaria en Pérez Zeledón". (p. I6). San José.

Consejo Nacional de Rectores (20l I). Estado de la Nación. Tercer Informe Programa Estado de la Educación. Consejo Nacional de Rectores, San José, Costa Rica.

Consejo Nacional de Rectores (201 I). Estado de la Nación. XVII Informe Programa Estado de la Nación. Consejo Nacional de Rectores, San José, Costa Rica.

Lebel, J. (2005). Salud, un enfoque ecosistémico. Bogotá: Alfaomega.

Ley Orgánica del Ambiente 7554 del 4 de octubre de 1995. Recuperado de http://www.webdelambiente.com/ legislacion/

Ministerio de Ambiente, Energía y Minas. (1998). Ley de Biodiversidad. San José. Recuperado de http://www. webdelambiente.com/legislacion/

Ministerio de Educación Pública. (2002). Plan de acción de educación para todos. (p. 14). San José: Ministerio de Educación Pública. www.oei.es/quipu/costarica/ PLANDEACCION2003_20I5.pdf

Ministerio de Salud. (2010). Plan Nacional de Salud para 2010-2021. (pp 54 y 78). San José: Ministerio de Educación Pública.

República de Costa Rica. (1994). Reforma Constitucional 7412 de 3 de junio de 1994. Constitución Política de Costa Rica. San José: Imprenta Nacional. 